\title{
Recurrence of Random Walks in the Ising Spins
}

Munemi Miyamoto

Yoshida College, Kyoto University, Kyoto, Japan

Abstract. Consider the $1 / 2$-Ising model in $Z^{2}$. Let $\sigma_{j}$ be the spin at the site $(j, 0) \in Z^{2}(j=0, \pm 1, \pm 2, \ldots)$. Let $\left\{X_{n}\right\}_{n=0}^{+\infty}$ be a random walk with the random transition probabilities such that

$$
P\left(X_{n+1}=j \pm 1 \mid X_{n}=j\right)=p_{j}^{ \pm} \equiv 1 / 2 \pm v\left(\sigma_{j}-\mu\right) / 2 .
$$

We show a case where $E\left[p_{j}^{+}\right] \geqq E\left[p_{j}^{-}\right]$, but $\lim _{n \rightarrow \infty} X_{n}=-\infty$ a.s. or $X_{n}$ is recurrent a.s.

Let $\left\{\sigma_{j}\right\}_{j=-\infty}^{+\infty}$ be an ergodic random sequence of \pm 1 spins with the mean $E\left[\sigma_{j}\right]=m$. Considering $-\sigma_{j}$ if $m<0$, we may assume $0 \leqq m<1$. Let $\left\{X_{n}\right\}_{n=0}^{+\infty}$ be a random walk with random transition probabilities such that

$$
\begin{aligned}
& P\left(X_{n+1}=j+1 \mid X_{n}=j\right)=p_{j}^{+} \equiv 1 / 2+v\left(\sigma_{j}-\mu\right) / 2, \\
& P\left(X_{n+1}=j-1 \mid X_{n}=j\right)=p_{j}^{-} \equiv 1 / 2-v\left(\sigma_{j}-\mu\right) / 2,
\end{aligned}
$$

where $v$ and $\mu$ are constants with

$$
|v|(1+|\mu|)<1 .
$$

We are interested in the recurrence of the random walk $\left\{X_{n}\right\}_{n=0}^{+\infty}$. Since the recurrence is trivial if $v=0$, let us assume $v \neq 0$. We apply Chung's results, which are summarized in the following

Lemma 1 (Sect. 12, Part I in [1]). Let $\left\{X_{n}\right\}_{n=0}^{+\infty}$ be a random walk with non-random positive transition probabilities $p_{j}^{ \pm}\left(p_{j}^{+}+p_{j}^{-}=1\right)$ which depend on $j$, i.e.,

$$
P\left(X_{n+1}=j \pm 1 \mid X_{n}=j\right)=p_{j}^{ \pm} .
$$

i) If $\sum_{r=1}^{+\infty} p_{1}^{-} p_{2}^{-} \ldots p_{r}^{-} /\left(p_{1}^{+} p_{2}^{+} \ldots p_{r}^{+}\right)=\sum_{r=-\infty}^{0} p_{r}^{+} p_{r+1}^{+} \ldots p_{0}^{+} /\left(p_{r}^{-} p_{r+1}^{-} \ldots p_{0}^{-}\right)=+\infty$, then $\left\{X_{n}\right\}_{n=0}^{+\infty}$ is recurrent a.s. 
ii) If $\sum_{r=1}^{+\infty} p_{1}^{-} p_{2}^{-} \ldots p_{r}^{-} /\left(p_{1}^{+} p_{2}^{+} \ldots p_{r}^{+}\right)<+\infty$ and

$\sum_{r=-\infty}^{0} p_{r}^{+} p_{r+1}^{+} \ldots p_{0}^{+} /\left(p_{r}^{-} p_{r+1}^{-} \ldots p_{0}^{-}\right)=+\infty$, then $\lim _{n \rightarrow+\infty} X_{n}=+\infty$ a.s.

iii) If $\sum_{r=1}^{+\infty} p_{1}^{-} p_{2}^{-} \ldots p_{r}^{-} /\left(p_{1}^{+} p_{2}^{+} \ldots p_{r}^{+}\right)=+\infty$ and

$\sum_{r=-\infty}^{0} p_{r}^{+} p_{r+1}^{+} \ldots p_{0}^{+} /\left(p_{r}^{-} p_{r+1}^{-} \ldots p_{0}^{-}\right)<+\infty$, then $\lim _{n \rightarrow+\infty} X_{n}=-\infty$ a.s.

In a case when transition probabilities are random as in ours, Lemma 1 shows that the condition

$$
E\left[\log \left(p_{j}^{-} / p_{j}^{+}\right)\right]=0
$$

is critical $[5,6]$. If $p_{j}^{ \pm}=1 / 2 \pm v\left(\sigma_{j}-\mu\right) / 2$, it is easy to see that

$$
\begin{aligned}
p_{j}^{-} / p_{j}^{+} & =A_{v}(\mu)^{1 / 2} B_{v}(\mu)^{-\sigma_{j} / 2} \\
& =\exp \left[-\left\{\sigma_{j}-\log A_{v}(\mu) / \log B_{v}(\mu)\right\} \log B_{v}(\mu) / 2\right],
\end{aligned}
$$

where

$$
\begin{aligned}
& A_{v}(\mu)=\left\{(1+v \mu)^{2}-v^{2}\right\} /\left\{(1-v \mu)^{2}-v^{2}\right\}, \\
& \left.B_{v}(\mu)=\left\{(1+v)^{2}-v^{2} \mu^{2}\right\} /\{1-v)^{2}-v^{2} \mu^{2}\right\} .
\end{aligned}
$$

Concerning condition (1), we have

Lemma 2. The equation for $\mu$

$$
A_{v}(\mu)=B_{v}(\mu)^{m}
$$

which is equivalent to (1), has a unique solution $\mu=\mu_{v}(m)$ in an interval $\left(-|v|^{-1}+1,|v|^{-1}-1\right)$.

For this $\mu_{\nu}(m)$, it holds that

$\mu_{v}(m)=\mu_{-v}(m)$,

$\mu_{v}(0)=0$,

$0<\mu_{v}(m)<m$, if $m>0$,

$\mu_{v}(m)$ is strictly monotone increasing in $m$.

We say that the sequence $\left\{\sigma_{j}\right\}_{j=-\infty}^{+\infty}$ generates weakly recurrent partial sums, if almost surely

$$
\begin{aligned}
& \frac{\lim }{n \rightarrow+\infty} \sum_{j=1}^{n}\left(\sigma_{j}-m\right), \quad \frac{\lim }{n \rightarrow-\infty} \sum_{j=n}^{0}\left(\sigma_{j}-m\right) \lessgtr \infty, \\
& \varlimsup_{n \rightarrow+\infty} \sum_{j=1}^{n}\left(\sigma_{j}-m\right), \quad \varlimsup_{n \rightarrow-\infty} \sum_{j=n}^{0}\left(\sigma_{j}-m\right) \supsetneqq-\infty .
\end{aligned}
$$

Our aim is to prove the following

Theorem. Assume that $\left\{\sigma_{j}\right\}_{j=-\infty}^{+\infty}$ generates weakly recurrent partial sums.

i) If $1-|v|^{-1}<\mu<\mu_{v}(m)$, then $\lim _{n \rightarrow+\infty} X_{n}=(\operatorname{sgn} v) \infty$ a.s.

ii) If $\mu=\mu_{v}(m)$, then $X_{n}$ is recurrent a.s.

iii) If $\mu_{v}(m)<\mu<|v|^{-1}-1$, then $\lim _{n \rightarrow+\infty} X_{n}=-(\operatorname{sgn} v) \infty$ a.s. 
Remark. Assume $m>0, v>0$. Then, $\mu_{v}(m) \leqq \mu<m$ implies $E\left[p_{j}^{+}\right]>E\left[p_{j}^{-}\right]$, i.e., the probability $p_{j}^{+}$that $X_{n}$ steps to the right is greater in the mean than the probability $p_{j}^{-}$to the left. But, our Theorem says that in this case $X_{n}$ is recurrent or $\lim _{n \rightarrow+\infty} X_{n}=-\infty$ according as $\mu=\mu_{v}(m)$ or $\mu_{v}(m)<\mu<m$.

Let $\sigma_{j}$ be the spin at $(j, 0) \in Z^{d}$ in the ferromagnetic Ising model in $Z^{2}$ with the nearest neighbour interactions. Let the probability measure $P$ be the limiting Gibbs distribution with the + boundary conditions. Then, all the assumptions on $\left\{\sigma_{j}\right\}_{j=-\infty}^{+\infty}$ in our Theorem are satisfied by this $\left\{\sigma_{j}\right\}_{j=-\infty}^{+\infty}$, i.e., we have

Proposition. The sequence of the Ising spins $\left\{\sigma_{j}\right\}_{j=-\infty}^{+\infty}$ stated above generates weakly recurrent partial sums.

Let us prove our results. At first we carry out

Proof of Lemma 2. In case $m=0, \mu=0$ is the unique solution of (2). Since $A_{-v}(\mu)$ $=A_{v}(\mu)^{-1}$, and $B_{-v}(\mu)=B_{v}(\mu)^{-1}$, we may assume $v>0$ and $m>0$.

Put

$$
\begin{aligned}
F_{v}(\mu)= & \left\{(1-v)^{2}-v^{2} \mu^{2}\right\}^{m} /\left\{(1-v \mu)^{2}-v^{2}\right\} \\
& -\left\{(1+v)^{2}-v^{2} \mu^{2}\right\}^{m} /\left\{(1+v \mu)^{2}-v^{2}\right\} \\
= & (1-v+v \mu)^{m} /\left\{(1-v-v \mu)^{1-m}(1-v \mu+v)\right\} \\
& -\left\{(1+v)^{2}-v^{2} \mu^{2}\right\}^{m} /\left\{(1+v \mu)^{2}-v^{2}\right\} .
\end{aligned}
$$

Equation (2) is equivalent to $F_{v}(\mu)=0$. It is easy to see that $F_{v}(\mu)$ is monotone increasing in $\mu \in\left[0, v^{-1}-1\right)$ and that

$$
F_{v}(0)<0, \quad F\left(v^{-1}-1-0\right)=+\infty .
$$

Therefore, (2) has a unique solution in $\left(0, v^{-1}-1\right)$. Since $A_{v}(\mu)<1$ and $B_{v}(\mu)>1$ for $\mu<0$, (2) has no negative solution.

From $A_{-v}(\mu)=A_{v}(\mu)^{-1}$ and $B_{-v}(\mu)=B_{v}(\mu)^{-1}$, it follows that $\mu_{v}(m)=\mu_{-v}(m)$.

Differentiating $\log A_{v}\left(\mu_{v}(m)\right)-m \log B_{v}\left(\mu_{v}(m)\right) \equiv 0$ in $m$, we have

$$
4 v\left\{1-v^{2}\left(\mu^{2}+2 m \mu+1\right)\right\} \mu^{\prime} /\left[\left\{(1+v \mu)^{2}-v^{2}\right\}\left\{(1-v \mu)^{2}-v^{2}\right\}\right]=\log B_{v}(\mu) \text {. }
$$

Since $v(1+|\mu|)<1$ and $v \log B_{v}(\mu)>0$, we have $\frac{d \mu_{v}(m)}{d m}>0$, i.e., $\mu_{v}(m)$ is strictly monotone increasing in $m$.

Let us prove $\mu_{v}(m)<m$ for $0<m<1$. If $m \geqq v^{-1}-1$, then $\mu_{v}(m)<v^{-1}-1 \leqq m$. Assume $m<v^{-1}-1$, i.e., $v<(m+1)^{-1}$. Let us introduce a function

We have

$$
G(v)=\log \left\{A_{v}(m) B_{v}(m)^{-m}\right\} \text { for } 0<v<(m+1)^{-1} \text {. }
$$

$$
\begin{gathered}
\frac{d G(v)}{d v}=8 m\left(1-m^{2}\right) v^{2} /\left[\left\{(1+v m)^{2}-v^{2}\right\}\left\{(1-v m)^{2}-v^{2}\right\}\right]>0, \\
\left(0<v<(m+1)^{-1}\right) .
\end{gathered}
$$

On the other hand, $G(0)=0$, hence $G(v)>0$, i.e., $A_{v}(m)>B_{v}(m)^{m}$. Therefore, $F_{v}(m)>0$. Since $F_{v}(\mu)$ is monotone increasing, we have $\mu_{v}(m)<m$. 
Proof of Theorem. Assume $v>0$.

1) Let $\mu \neq \mu_{v}(m)$. We have

$$
\begin{aligned}
& p_{1}^{-} p_{2}^{-} \ldots p_{r}^{-} /\left(p_{1}^{+} p_{2}^{+} \ldots p_{r}^{+}\right) \\
& \quad=\exp \left[-\left\{\log B_{v}(\mu) / 2\right\} \sum_{j=1}^{r}\left\{\sigma_{j}-\log A_{v}(\mu) / \log B_{v}(\mu)\right\}\right] .
\end{aligned}
$$

Since $\sum_{j=1}^{r}\left\{\sigma_{j}-\log A_{v}(\mu) / \log B_{v}(\mu)\right\} \sim r\left\{m-\log A_{v}(\mu) / \log B_{v}(\mu)\right\} \quad$ as $r \rightarrow+\infty$ by the point-wise ergodic theorem, we have

$$
\sum_{r=1}^{\infty} p_{1}^{-} p_{2}^{-} \ldots p_{r}^{-} /\left(p_{1}^{+} p_{2}^{+} \ldots p_{r}^{+}\right)\left\{\begin{array}{l}
<+\infty, \text { if } m-\log A_{v}(\mu) / \log B_{v}(\mu)>0, \\
=+\infty, \text { if } m-\log A_{v}(\mu) / \log B_{v}(\mu)<0
\end{array}\right.
$$

Our results in case $\mu \neq \mu_{v}(m)$ follow from Lemma 1.

2) Let $\mu=\mu_{v}(m)$. We have

$$
p_{1}^{-} p_{2}^{-} \ldots p_{r}^{-} /\left(p_{1}^{+} p_{2}^{+} \ldots p_{r}^{+}\right)=\exp \left[-\left\{\log B_{v}(\mu) / 2\right\} \sum_{j=1}^{r}\left(\sigma_{j}-m\right)\right] \text {. }
$$

Since $\left\{\sigma_{j}\right\}$ generates weakly recurrent partial sums, the sequence $\sum_{j=1}^{r}\left(\sigma_{j}-m\right)$ hits a bounded set infinitely often as $r \rightarrow+\infty$. Therefore

$$
\sum_{r=1}^{+\infty} p_{1}^{-} p_{2}^{-} \ldots p_{r}^{-} /\left(p_{1}^{+} p_{2}^{+} \ldots p_{r}^{+}\right)=+\infty \quad \text { a.s. }
$$

Our results also follow from Lemma 1.

Let us proceed to the

Proof of Proposition. Let $\beta$ and $h$ be the reciprocal temperature and the external field, respectively.

1) Case $\beta \leqq \beta_{c}$ and $h=0$. In this case, $m=0$. Suppose

$$
p\left(\lim _{n \rightarrow+\infty} \sum_{j=1}^{n} \sigma_{j}=+\infty\right)>0 .
$$

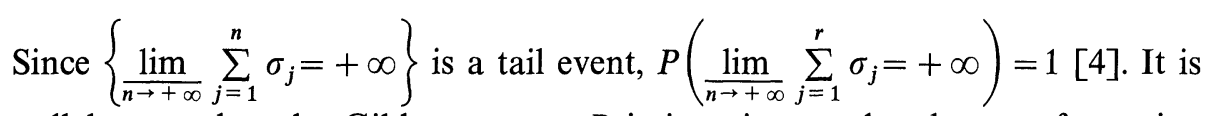
well known that the Gibbs measure $P$ is invariant under the transformation $\sigma_{x} \rightarrow-\sigma_{x}\left(x \in Z^{2}\right)$. Therefore, $P\left(\varlimsup_{n \rightarrow+\infty} \sum_{j=1}^{n} \sigma_{j}=-\infty\right)=1$, which is a contradiction. Hence, $\lim _{n \rightarrow+\infty} \sum_{j=1}^{n} \sigma_{j}<+\infty$ a.s.

2) Case $\beta>\beta_{c}$ or $h \neq 0$. Since the correlations decay exponentially in this case, condition (3) in the following Lemma 3 holds for $\left\{\sigma_{n}\right\}$ in place of $\left\{\xi_{n}\right\}$ ([2]). Therefore, our result in Proposition is a corollary to 
Lemma 3. Let $\left\{\xi_{n}\right\}_{n=-\infty}^{+\infty}$ be a stationary sequence of bounded random variables with $E\left[\xi_{n}\right]=0$. For $n<m$, let $\mathscr{B}_{n}^{m}$ be the $\sigma$-algebra generated by $\left\{\xi_{j} ; n \leqq j \leqq m\right\}$.Put

$$
\alpha(n)=\sup \left\{|P(A \cap B)-P(A) P(B)| ; A \in \mathscr{B}_{-\infty}^{0}, B \in \mathscr{B}_{n}^{+\infty}\right\} .
$$

If

$$
\sum_{n=1}^{+\infty} \alpha(n)<+\infty
$$

then $\varlimsup_{n \rightarrow+\infty} \sum_{j=1}^{n} \xi_{j}=+\infty$ and $\lim _{n \rightarrow+\infty} \sum_{j=1}^{n} \xi_{j}=-\infty$ a.s.

Proof. The central limit theorem holds for this $\left\{\xi_{n}\right\}$ [3], i.e.,

$$
\lim _{n \rightarrow+\infty} P\left(\sum_{j=1}^{n} \xi_{j} /(\sigma \sqrt{n})>z\right)=1 / \sqrt{2 \pi} \int_{z}^{+\infty} e^{-x^{2} / 2} d x
$$

where $\sigma^{2}=E\left[\xi_{0}^{2}\right]+2 \sum_{j=1}^{+\infty} E\left[\xi_{0} \xi_{j}\right]<+\infty$. Putting $z=1 / \sigma$, we can find $N_{0} \geqq 1$ such that for any $n \geqq N_{0}$

$$
P\left(\sum_{j=1}^{n} \xi_{j}>\sqrt{n}\right) \geqq \frac{1}{2} \frac{1}{\sqrt{2 \pi}} \int_{1 / \sigma}^{+\infty} e^{-x^{2} / 2} d x \equiv \delta .
$$

Let $c \geqq 1$ be a constant such that $\left|\xi_{n}\right| \leqq c$. Put $n_{1}=N_{0}$. A sequence $\left\{n_{k}, m_{k}\right\}_{k=1}^{+\infty}$ is defined recursively in the following way;

$$
\left\{\begin{array}{l}
m_{k}=n_{k}+k \\
n_{k+1}=m_{k}+\left(c m_{k}+k\right)^{2}
\end{array}\right.
$$

Remark that $\sqrt{n_{k+1}-m_{k}}=c m_{k}+k \geqq N_{0}$. Put

$$
E_{k}=\left\{\sum_{j=m_{k}+1}^{n_{k+1}} \xi_{j}>\sqrt{n_{k+1}-m_{k}}\right\} \text {. }
$$

We have

$$
\begin{aligned}
P\left(\bigcap_{k=K}^{+\infty} E_{k}^{c}\right) & =P\left(E_{K}^{c} \cap \bigcap_{k=K+1}^{+\infty} E_{k}^{c}\right) \\
& \leqq P\left(E_{K}^{c}\right) P\left(\sum_{k=K+1}^{+\infty} E_{k}^{c}\right)+\alpha(K+1) \\
& \leqq(1-\delta) P\left(\bigcap_{k=K+1}^{+\infty} E_{k}^{c}\right)+\alpha(K+1) .
\end{aligned}
$$

Letting $K \rightarrow+\infty$, we have $\lim _{K \rightarrow+\infty} P\left(\bigcap_{k=K}^{+\infty} E_{k}^{c}\right) \leqq(1-\delta) \lim _{K \rightarrow+\infty} P\left(\bigcap_{k=K}^{+\infty} E_{k}^{c}\right)$, hence

$$
P\left(\bigcup_{K=1}^{+\infty} \bigcap_{k=K}^{+\infty} E_{k}^{c}\right)=\lim _{K \rightarrow+\infty} P\left(\bigcap_{k=K}^{+\infty} E_{k}^{c}\right)=0 .
$$


Therefore, $P\left(\bigcap_{K=1}^{+\infty} \bigcup_{k=K}^{+\infty} E_{k}\right)=1$, i.e., infinitely many $E_{k}$, s occur a.s. If $E_{k}$ occurs, then

$$
\sum_{j=1}^{n_{k}+1} \xi_{j}=\sum_{j=1}^{m_{k}} \xi_{j}+\sum_{j=m_{k}+1}^{n_{k+1}} \xi_{j} \geqq-c m_{k}+\sqrt{n_{k+1}-m_{k}}=k .
$$

Thus, $\varlimsup_{n \rightarrow+\infty} \sum_{j=1}^{n} \xi_{j}=+\infty$ a.s.

\section{References}

1. Chung, K.L.: Markov chains with stationary transition probabilities. Berlin, Heidelberg, New York: Springer 1960

2. Hegerfeldt, G.C., Nappi, Ch.R.: Mixing properties in lattice systems. Commun. Math.Phys.53, 1-7 (1977)

3. Ibragimov, I.A., Linnik, Yu.V.: Independent and stationarily dependent variables (in Russian). Moscow: Nauka 1965

4. Miyamoto, M.: Martin-Dynkin boundaries of random fields. Commun. Math. Phys. 36, 321-324 (1974)

5. Sinai, Ya.G.: Limit behaviour of one-dimensional random walks in random environment (in Russian). Teor. Veroyatn. 27, 247-258 (1982)

6. Solomon, F.: Random walks in a random environment. Ann. Prob. 3, 1-31 (1975)

Communicated by Ya. G. Sinai

Received August 1, 1984 